$10-2013$

\title{
On the origin of mode- and bond-selectivity in vibrationally mediated reactions on surfaces
}

Daniel Killelea

dkillelea@e.luc.edu

Arthur L. Utz

Tufts University

Follow this and additional works at: https://ecommons.luc.edu/chemistry_facpubs

Part of the Atomic, Molecular and Optical Physics Commons, Materials Chemistry Commons, and the Physical Chemistry Commons

\section{Recommended Citation}

Killelea, Daniel and Utz, Arthur L.. On the origin of mode- and bond-selectivity in vibrationally mediated reactions on surfaces. Physical Chemistry Chemical Physics, 15, 47: 20545-20554, 2013. Retrieved from Loyola eCommons, Chemistry: Faculty Publications and Other Works, http://dx.doi.org/10.1039/ C3CP53765J

This Article is brought to you for free and open access by the Faculty Publications and Other Works by Department at Loyola eCommons. It has been accepted for inclusion in Chemistry: Faculty Publications and Other Works by an authorized administrator of Loyola eCommons. For more information, please contact ecommons@luc.edu. cc) (i) $\ominus$

This work is licensed under a Creative Commons Attribution-Noncommercial-No Derivative Works 3.0 License. (C) The Owner Societies, 2013. 
Cite this: Phys. Chem. Chem. Phys., 2013,

\title{
On the origin of mode- and bond-selectivity in vibrationally mediated reactions on surfaces
}

15, 20545

Received 4th September 2013, Accepted 22nd October 2013

DOI: $10.1039 / c 3 c p 53765 j$

www.rsc.org/pccp

\author{
Daniel R. Killelea*a and Arthur L. Utz*b
}

\begin{abstract}
The experimental observations of vibrational mode- and bond-selective chemistry at the gas-surface interface indicate that energy redistribution within the reaction complex is not statistical on the timescale of reaction. Such behavior is a key prerequisite for efforts to use selective vibrational excitation to control chemistry at the technologically important gas-surface interface. This paper outlines a framework for understanding the origin of non-statistical reactivity on surfaces. The model focuses on the kinetic competition between intramolecular vibrational energy redistribution (IVR) within the reaction complex, which in the long-time limit leads to statistical behavior, and quenching, scattering, or desorption processes that restrict the extent of IVR prior to reaction. Characteristic timescales for these processes drawn from studies of vibrational energy flow dynamics on surfaces and in the gas and condensed phases suggest that IVR is severely limited for important classes of surface reactions. Under these conditions, selective vibrational excitation can lead to preferential transition state access and result in mode- or bond-selective chemistry, even at high collision energies above the barrier to reaction. In addition to providing a basis for understanding experimental observations, the model provides guidance for identifying other gas-surface reactions that may exhibit mode-selective behavior.
\end{abstract}

\section{Introduction}

The study of chemical reaction dynamics reveals how nuclear motion and patterns of energy flow impact chemical reactivity. Using that knowledge to manipulate the yield or identity of reaction products is a long-standing goal. ${ }^{1}$ During the past decade, state-resolved gas-surface reactivity and adsorbate excitation studies ${ }^{2-4}$ have extended the realm of vibrational mode- and bond-selective chemistry from the gas phase to the technologically important gas-surface interface. There are now examples of vibrational mode selective surface chemistry, ${ }^{5-12}$ in which the identity of the reagent's vibrational state, and not just its energy, determines reaction probability, and bond selective surface chemistry ${ }^{13,14}$ where selective vibrational excitation leads to preferential cleavage of a particular chemical bond. Recent reviews provide a detailed summary of experimental methods and results. ${ }^{15-17}$ This article describes the molecular-level origin of these non-statistical reactivity patterns on surfaces, and it explores how this behavior might extend to increasingly larger chemical systems.

\footnotetext{
${ }^{a}$ Department of Chemistry and Biochemistry, Loyola University Chicago, 1068 W. Sheridan Rd., Chicago, IL 60660, USA. E-mail: dkillelea@luc.edu; Fax: +1 773508 3086; Tel: +1 7735083136

${ }^{b}$ Tufts University Department of Chemistry and W. M. Keck Laboratory for Materials Chemistry, 62 Talbot Ave., Medford, MA 02155, USA.

E-mail: arthur.utz@tufts.edu; Fax: +1617627 3443; Tel: +1 6176273473
}

The many facile electron- and phonon-mediated energy transfer channels available to a reaction complex at the gas-metal interface ${ }^{18}$ have led some authors to explore the suitability of statistical reactivity models for gas-surface reactions. Harrison and co-workers adapted microcanonical unimolecular rate theory to describe the dissociative chemisorption of methane and other small molecules on surfaces. ${ }^{19-23}$ By adjusting the number and frequency of surface phonon modes that participate in reaction and neglecting electronically non-adiabatic processes, they reproduced a wide range of internal-state-averaged bulb and beam-surface scattering data.

Statistical theories are useful benchmarks for experimental measurements of reaction dynamics. They relate a reaction's rate to the statistical probability that an amount of energy equal to or greater than its threshold energy appears in the reaction coordinate. When supplemented by dynamical constraints on energy flow within the reaction complex, these theories can provide valuable insight into the rate of reaction and the partitioning of excess energy among reaction products. ${ }^{24} \mathrm{~A}$ system will be welldescribed by a statistical theory when either of two conditions is met. ${ }^{25}$ In the first, rapid and complete intramolecular vibrational energy redistribution (IVR) within the reaction complex ensures that each reaction complex is equally likely to appear in any of the energetic configurations in a microcanonical ensemble. In this scenario, even molecules prepared in select quantum states would react statistically because once complete, IVR would 
randomize the energy distribution prior to reaction. In the second, IVR could be much slower than the timescale of reaction, but if all possible energetic configurations are statistically represented within the ensemble of reactants, then the ensemble-averaged reactivity still reflects the statistical limit. While both scenarios result in ensemble-averaged reactivity patterns consistent with a statistical model, the distinction is crucial for understanding how to manipulate chemistry. Controlling the outcome of a reaction by selective vibrational excitation only succeeds if IVR is incomplete on the reaction timescale.

Experiments that measure the reactivity of thermally averaged ensembles of molecules cannot differentiate these cases, but internal-state-resolved studies can. State-resolved gas-surface scattering experiments have quantified reactivity for thirteen unique vibrational state/surface structure combinations of methane dissociation on metals..$^{2,3,5-10,12-16,26-28}$ In addition, Beck and co-workers compared activation for a precursormediated reaction by two different vibrational states of silane on $\operatorname{Si}(100)(1 \times 2) .{ }^{15}$ Thirteen of these fifteen systems exhibited clear signatures of mode-selective behavior. In a separate study of $\mathrm{CHD}_{3}$ dissociation on $\mathrm{Ni}(111)$, excitation of one quantum of $\mathrm{C}-\mathrm{H}$ stretch in the gas-phase reagent led to a more than 90 -fold enhancement in the relative yield of $\mathrm{C}-\mathrm{H}$ bond cleavage products. ${ }^{13}$ Pronounced bond-selectivity has also been reported for $\mathrm{CHD}_{3}, \mathrm{CH}_{2} \mathrm{D}_{2}$, and $\mathrm{CH}_{3} \mathrm{D}$ on $\mathrm{Pt}(110)-(1 \times 2) .{ }^{14}$ Taken together, these studies clearly show that vibrational energy redistribution is not complete before reaction, and that mode- or bond-selective behavior must be considered when describing the reactivity of polyatomic molecules on surfaces.

Here, we survey the general features of chemical systems that permit vibrationally mediated control of surface reactions and move beyond the question of whether or not a fully statistical theory is applicable to surface reactions. We consider processes that occur on the ground electronic state because they influence the dynamics of all gas-surface encounters. Recent observations suggest that electronically non-adiabatic processes also play a role in the dynamics of surface reactions. ${ }^{29,30}$ We address this issue by including in our framework efficient vibrational quenching, which may involve electron-hole pair excitation, as a kinetic process competing with reaction. We draw from recent studies of vibrational energy flow in the gas-phase and solution and identify guiding principles relevant to a diverse range of chemical systems. We then extend those ideas to vibrationally mediated reactions at the gas-surface interface to better understand experimental trends and identify strategies that exploit energy flow kinetics to manipulate or control reactivity.

\section{Results and discussion}

For a system to exhibit mode- or bond-selective reactivity, the system must provide preferential transition state access for a particular vibrational motion, and the vibrational character of the prepared state must survive or evolve in a way that exploits that bias. Within the context of the Born-Oppenheimer approximation, a multidimensional potential energy surface (PES) governs reactivity. When reagents are distorted from their equilibrium geometry at the transition state (a "late" barrier), Polanyi's rule suggests that vibrational excitation in the deformation coordinate will enhance reactivity and provide the necessary bias. ${ }^{31}$ Once a bias for transition state access exists, the nature of the prepared state, energy flow processes that redistribute that excitation, and the timescales for these processes relative to reaction determine whether a particular vibration will exhibit mode-or bond-selective reactivity. It is these energy flow processes that complicate the simple extension of Polanyi's rules to reactions of polyatomic molecules. ${ }^{32}$

Normal modes are often used to describe the nuclear motions of polyatomic molecules. These states are the solutions to a model Hamiltonian that contains only diagonal harmonic terms in the potential energy operator. They form a complete zero-order basis set for describing the molecule's vibrational states. The full molecular Hamiltonian, when expressed in the basis of normal mode states, contains higher-order anharmonic coupling and vibration-rotation interaction terms that are present in the real molecule but absent from the harmonic oscillator approximation. These additional terms couple the zero-order normal mode states and result in a new basis set of vibrations that are the rovibrational eigenstates of the molecule. The vibrational identity of these eigenstates is time-independent. Each eigenstate corresponds to periodic vibrational motion of the molecule's nuclei that does not evolve until interrupted by collision or radiative decay.

Fig. 1 illustrates the relationship between the zero-order basis states and the molecular eigenstates for a small polyatomic molecule, expressed in the context of the infrared excitation step of a state-resolved beam-surface scattering experiment. In the two-state limit shown in Fig. 1a, state $|s\rangle$ represents a "bright" zero order state (e.g. a $\mathrm{C}-\mathrm{H}$ stretch) whose vibrational character leads to strong oscillator strength for an excitation transition of interest. State $\{|\mathrm{I}\rangle\}$ is a zero-order "dark" state (e.g. an overtone or combination of low frequency bending modes) whose energy is similar to that of state $|s\rangle$, but whose oscillator strength is much less than that of $|s\rangle$. The Hamiltonian in the zero-order basis includes terms in the kinetic and potential energy operators that couple the zero-order states to form the set of molecular eigenstates, $\{|n\rangle\}$. The strength of this coupling is greatest when

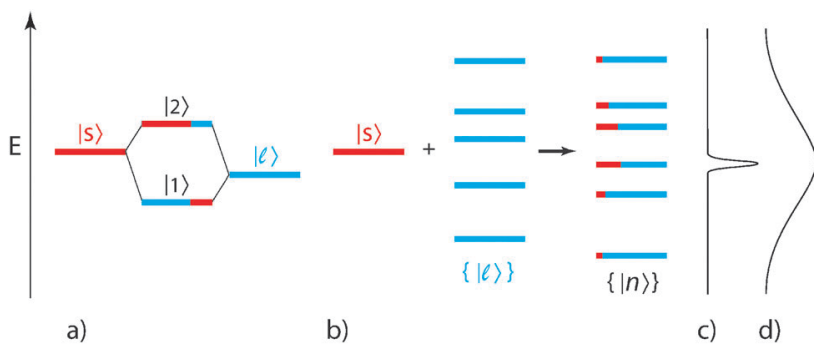

Fig. 1 Zero-order state mixing. In panel (a), mixing of the bright $(|s\rangle$, red) and

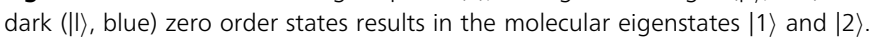
Fig. $1 \mathrm{~b}$ shows the case for a bright state mixing with a sparse bath of dark states to form the set of molecular eigenstates $\{|n\rangle\}$. The energy spread of a narrow and broadband excitation source relative to the eigenstate spacing is illustrated in (c) and (d), respectively. 
the interacting zero-order states (1) differ by a small number of vibrational quanta, (2) are nearly degenerate in energy, and (3) are coupled by significant off-diagonal elements in the Hamiltonian matrix. The number of quanta by which the states differ is often referred to as the coupling order. For example, a $\mathrm{C}-\mathrm{H}$ stretching fundamental $(v=1)$ and the $\mathrm{H}-\mathrm{C}-\mathrm{H}$ bending overtone $(v=2)$ differ by a total of three quanta and are therefore coupled by a thirdorder cubic anharmonic potential term $q_{\mathrm{s}} q_{\mathrm{b}},{ }^{2}$ where $q_{\mathrm{s}}$ and $q_{\mathrm{b}}$ are the stretching and bending coordinates. High order coupling is generally very weak due to the vanishing magnitude of very high order terms in the Hamiltonian.

Depending on the extent of intramolecular coupling in the zero-order basis, the eigenstates may or may not closely resemble the normal mode vibrations. ${ }^{33}$ Fig. 1 b shows how a single bright state interacts with a sparse bath of dark states. Bright state character is diluted among the eigenstates $\{|n\rangle\}$ according to the degree of coupling among the states. The intensity of the excitation transition depends on the amount of "bright" zeroorder state character in the eigenstate. High quality anharmonic force field calculations, such as those reported for $\mathrm{CH}_{4},{ }^{34}$ reveal the extent of zero-order state mixing and the normal mode basis set composition of each eigenstate in the molecule.

The vibrational eigenstates form a palette of states available for optical or inelastic electron tunnelling excitation. When the energy resolution of the excitation source is narrower than the spacing between eigenstates, as shown in Fig. 1c, only one eigenstate state is resonant with the light source and excitation prepares the molecule in a single vibrational eigenstate. The vibrational character of that state does not evolve in time. Excitation with a broader energy source prepares a coherent superposition of eigenstates. In the limit where the excitation source is broad enough in energy to coherently excite all eigenstates containing bright zero-order state character $(|s\rangle)$, as shown in Fig. 1d, excitation prepares the zero-order state at $t=0$. Since each of the constituent eigenstates in this superposition state has different vibrational energies, their vibrational frequencies differ. Their nuclear displacements therefore diphase with time, resulting in a time-dependent evolution in the vibrational motion of the molecule, or IVR.

This palette of vibrational states available for excitation may or may not include a vibration that projects directly along the reaction coordinate. For example, in the transition state for methane's dissociative chemisorption on $\mathrm{Ni}(111),{ }^{35-40}$ only one $\mathrm{C}-\mathrm{H}$ bond is significantly extended, but the infrared- and Raman-active vibrations available for gas phase excitation are collective excitations of all four $\mathrm{C}-\mathrm{H}$ oscillators. Isotopic substitution can change the identity of the molecule's normal mode vibrations and vibrational eigenstates, and is therefore a useful tool for expanding the range of vibrational motions available for probing transition state access. Surface bound molecules frequently have vibrational eigenstates that differ from those of the isolated gas phase molecule, so the decision to excite a reagent before or after adsorption also impacts the identity of the initially prepared state.

Two experimental approaches have produced vibrationalstate-resolved measurements of reactivity at the gas-surface interface. ${ }^{15-17}$ In the first, high-resolution infrared ${ }^{4,41,42}$ or
Raman $^{43}$ excitation of molecules in the collision-free region of a supersonic molecular beam prepares a single rovibrational eigenstate of the isolated gas-phase molecule, as depicted in Fig. 1c. Because the excited molecules are in a pure eigenstate (stationary state), IVR does not occur. Furthermore, the radiative lifetime of methane vibrations is significantly longer than the 100-200 $\mu$ s flight time from excitation region to the surface, so reagents approach the surface in their initially prepared state with a well-defined translational energy $\left(E_{\text {trans }}\right)$.

In the second approach, inelastic electron tunnelling or optical excitation excites a vibrational mode of a reagent already adsorbed to a surface. ${ }^{11,44}$ The adsorbate-surface complex has a high density of states dominated by low frequency frustrated translations and rotations, the adsorbate-surface stretch, and surface atom (phonon) vibrations. These "dark" zero-order states mix with the "bright" state chromophore that is typically centered in the adsorbate and form a set of eigenstates for the adsorbate-surface complex. Under these conditions, the bandwidth of the excitation source is frequently broader than the spacing between states, and excitation produces a coherent superposition state. Immediately following excitation, the coherently excited eigenstates beat against each other, resulting in IVR as the vibrational character of the adsorbate-surface complex evolves in time. The nuclear motion of vibrations prepared in this way will generally differ from those of a gas-phase molecule. The prepared state may include contributions from substrate atom motion and frustrated translations or rotations. A more thorough discussion of these two approaches to state preparation appears in ref. 15.

For experiments using the first preparation approach, where narrow bandwidth excitation of reagents in a molecular beam prepares a single eigenstate of the gas phase molecule, IVR can still occur prior to reaction because the pure gas-phase eigenstate is altered by the molecule's proximity to the metal surface. As the molecule draws within several $\AA$ of the surface, potential energy terms in the Hamiltonian that describe the moleculesurface interaction are no longer negligible. Those terms alter the Hamiltonian, and the system's vibrational eigenstates evolve from those of an isolated gas-phase reagent and surface to those of the interacting molecule-surface complex, as illustrated pictorially in Fig. 2. This evolution of eigenstate identity has important repercussions for vibrational energy flow. Even though the laser prepared state was an eigenstate of the isolated gas phase molecule, it is not an eigenstate of the molecule-surface complex. Instead, the laser-prepared state becomes a superposition state within the basis of molecule-surface complex eigenstates. Timedependent vibrational energy flow results as the newly formed superposition state dephases. In other words, the molecule's proximity to the surface initiates and induces the IVR process within the molecule-surface complex. The potential energy of interaction between molecule and surface depends on orientation, distance, and impact site, so the Hamiltonian and its associated eigenstates continue to evolve and drive further IVR as the molecule approaches the surface. This process is captured well by reaction path Hamiltonian computational methods that have recently shown great promise for capturing the essential dynamics of 


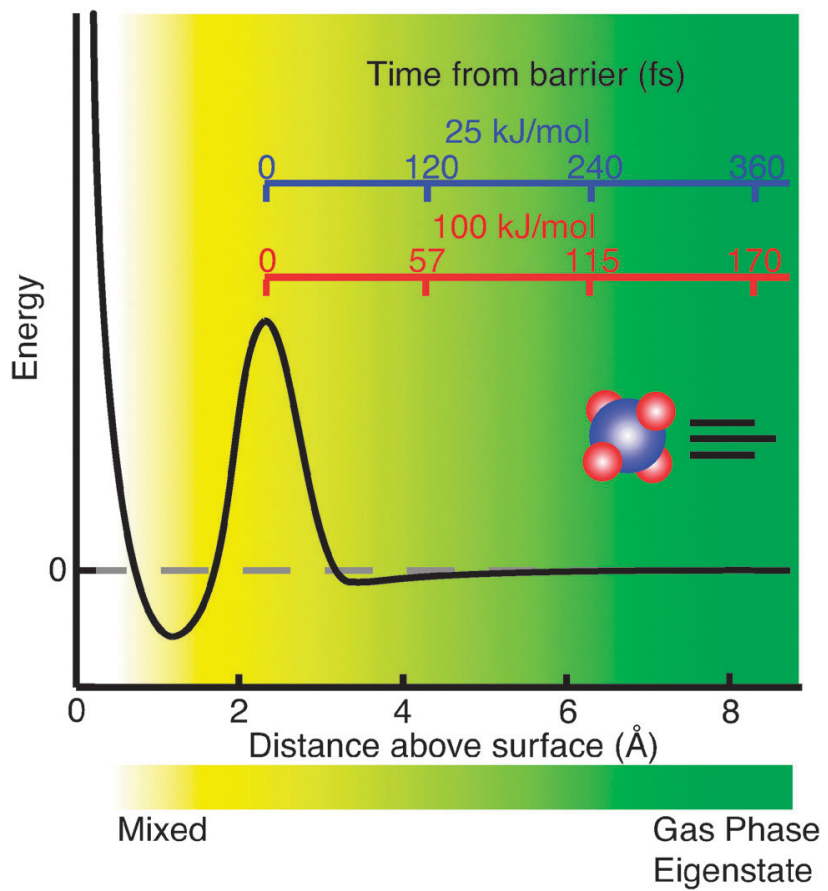

Fig. 2 Surface-induced IVR. As an incident methane molecule nears the surface, the gas-surface interaction potential alters the identity of the system's vibrational eigenstates. The green to yellow gradient illustrates the evolution of the molecule's vibrational state identity as the molecule approaches and is perturbed by the surface. On the right, the initially prepared state is an eigenstate of the isolated gas-phase molecule. As it approaches the surface, that state becomes a mixed superposition of molecule-surface eigenstates. Because of its velocity, there is less than one picosecond between when the molecule first begins to experience the gas-surface potential and when it encounters the energetic barrier to reaction, as shown for two incident translational energies at the top of the figure.

methane activation on metal surfaces. ${ }^{45-47}$ The detailed pathways and rates of IVR in a particular molecule depend on the nature and magnitude of the coupling terms in the gas-surface potential and on the molecule's trajectory.

Mode- and bond-selective chemistry is not possible if extensive IVR occurs prior to reaction. In the case where the approach to the surface is extremely slow (nearly zero velocity), complete statistical redistribution of energy throughout all accessible states might be expected. However, when the time available for IVR is limited, then non-statistical behavior will be most pronounced. Surface residence time and vibrational quenching compete with collision-induced IVR and are the primary factors limiting IVR in beam-surface scattering experiments, as shown in Fig. 2. Molecules approach the surface at speeds greater than $1000 \mathrm{~m} \mathrm{~s}^{-1}$ and only begin to experience the gas-surface potential a few $\AA$ from the surface, leaving only a few 100's of femtoseconds for IVR to occur and influence the reagent's trajectory at the energetic barrier to reaction. Quenching by phonon or electronically mediated mechanisms removes energy ( $\left.E_{\mathrm{vib}}, E_{\text {trans }}\right)$ from the molecule and further limits the time available for IVR. Because few experiments to date have provided detailed insight into the IVR dynamics of adsorbed polyatomic molecules on metal surfaces, we turn to results from the gas phase and solution for guidance on the rates and pathways of IVR in chemical systems with a high vibrational state density.

Elles et al. used broadband infrared laser excitation (cf. Fig. 1c) followed by UV absorption to study IVR from the $v=2$ zero-order $\mathrm{C}-\mathrm{H}$ stretch in $\mathrm{CH}_{3} \mathrm{I}$ into the Franck-Condon active C-I stretch in the gas-phase and in solution. ${ }^{48,49}$ Their gas-phase measurements revealed bimodal energy flow kinetics from the $\mathrm{C}-\mathrm{H}$ to the $\mathrm{C}-\mathrm{I}$ stretch, with a fast (5-10 ps) and slow (100's of ps) channels. A tiered model of IVR first proposed by Sibert et $a .^{50}$ and based on earlier work by Stannard and Gelbart $^{51}$ explains their observations. This model expands on the simple bright/dark state model of Fig. 1 to better describe IVR in systems with a high vibrational state density. Because the initially prepared zero-order $\mathrm{C}-\mathrm{H}$ stretch state is not an eigenstate, off-diagonal matrix elements in the full molecular Hamiltonian couple it to other vibrational states, as shown in Fig. 3. The initial (fast) step of IVR corresponds to energy flow from the C-H stretch into a relatively small number of "doorway" vibrational states. Doorway states are those most strongly coupled to the initially excited state via anharmonic or other low-order (small $\Delta v$ ) intramolecular coupling mechanisms. ${ }^{52}$

The slower component of IVR corresponds to relaxation of the doorway states into subsequent tiers of states to which they are coupled. This stepwise process continues until energy is fully redistributed throughout the full density of states within the molecule. The model succeeds because step-wise energy flow via a sequence of low-order coupling steps is typically faster than parallel relaxation from the initial state into the full bath via inefficient high order coupling. ${ }^{49,53,54}$

Two features of the tier model have important implications for IVR dynamics. First, since the relaxation rate for the initial state is defined by its coupling strength and energy match with the doorway states, the initial IVR relaxation rate is largely independent of the molecule's full vibrational state density.

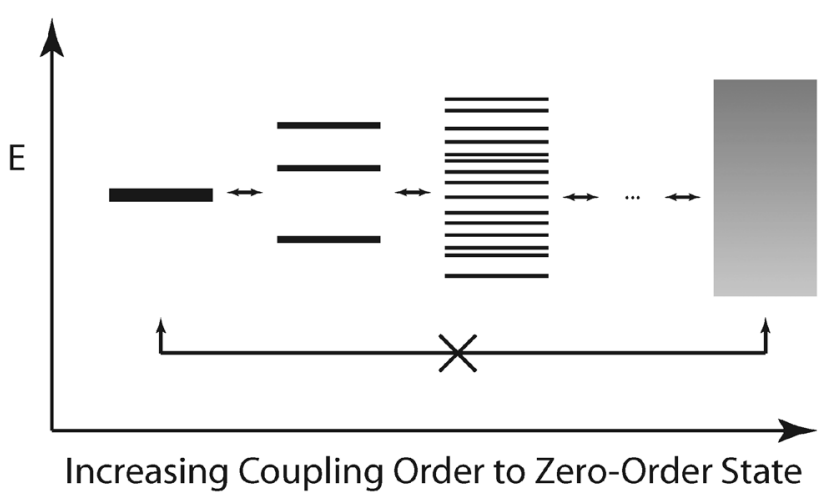

Fig. 3 Tiered model of vibrational energy flow. The initial state appears on the left. If it is not an eigenstate of the system, it is coupled to other vibrational states in the molecule. The small number of states most strongly coupled to the initial state form the first tier of coupled states. The first tier is in turn coupled, again by low-order coupling, to a second tier of states. This process continues through subsequent tiers until the entire bath of vibrational states is coupled. In general, energy flow via sequential low order coupling from one tier to the next is faster than direct relaxation from the initial state to the full bath via high order coupling. 


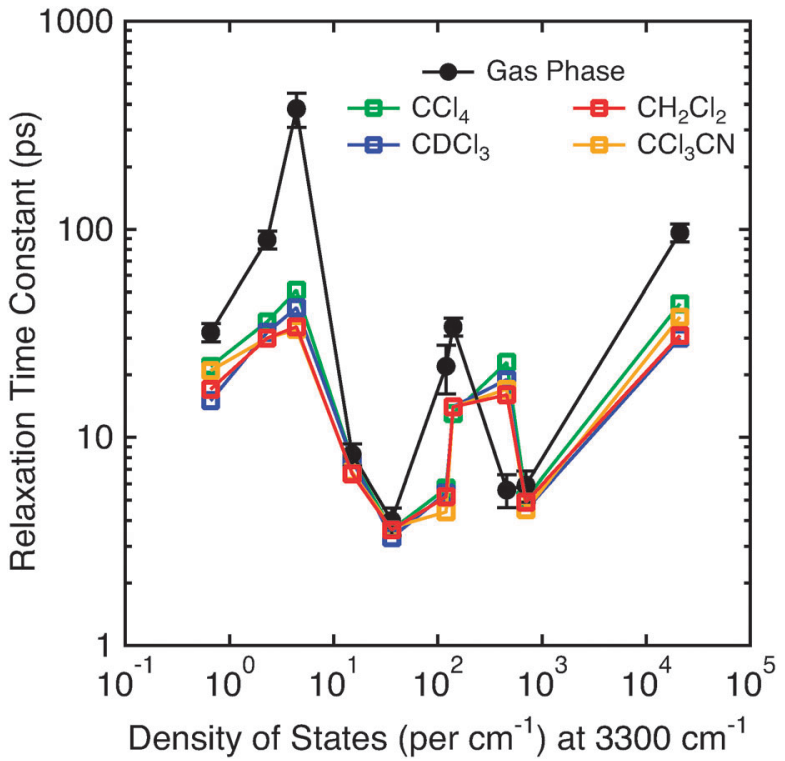

Fig. 4 Influence of molecular complexity and solvation on the initial rate of IVR. Data are from ref. 55-57. A series of acetylenic molecules whose vibrational state densities span nearly six orders of magnitude were prepared in $\mathrm{v}=1$ of the $\mathrm{C}-\mathrm{H}$ stretch. The time constant for the initial step of IVR in the gas phase shows no systematic dependence on molecular complexity. Upon solvation, the time constant for IVR is only modestly altered from the gas-phase value, and solvent polarity has a minimal effect.

Second, since relaxation through the tiers is sequential, complete relaxation into the full bath of energetically accessible states cannot be faster than the initial step.

Experimental work by Pate and co-workers ${ }^{55-57}$ supports the tier model. They followed the IVR dynamics of the zero-order acetylenic $\mathrm{C}-\mathrm{H}$ stretch in gas-phase molecules whose vibrational state density ranged from 0.6 to 20000 states $\mathrm{cm}^{-1}$. Despite the wide variation in the number of nearly resonant states in these molecules, time constants for the initial step of IVR, which are shown in Fig. 4, range from a few picoseconds to a few 100's of picoseconds and show no clear correlation with vibrational state density. Instead, the variation arises from subtle differences in molecular structure that tune and detune the resonance and coupling between the $\mathrm{C}-\mathrm{H}$ stretch state and the doorway states to which it is most strongly coupled.

When a molecule is in solution or adsorbed on a surface, its chemical environment provides and additional bath of vibrational states into which energy can flow. Comparing IVR rates for a molecule in the gas phase and in solution provides insight into how chemical environment might influence IVR. Elles et $a l^{48,49}$ found that the rate constant for IVR from the $v=2$ $\mathrm{C}-\mathrm{H}$ stretch in $\mathrm{CH}_{3} \mathrm{I}$ is essentially identical in the gas phase and in solution. Similarly, Pate and co-workers ${ }^{55-57}$ showed that solvents ranging from non-polar $\mathrm{CCl}_{4}$ to highly polar $\mathrm{CCl}_{3} \mathrm{CN}$ do not significantly alter the initial IVR decay rate of the acetylenic $\mathrm{C}-\mathrm{H}$ stretch $(v=1)$. The time constants, shown in Fig. 4, range from a few picoseconds up to nearly a nanosecond. Neither interactions with the solvent nor the introduction of a very high-density bath of solute-solvent vibrations significantly alter the initial IVR rate. Therefore, even for solvated molecules, the initial IVR rate of energy flow into the doorway states largely reflects the IVR dynamics of the isolated gas-phase molecule, and the fastest IVR processes occur on the picosecond timescale, even in chemically complex systems.

While the initial rates of IVR in the gas phase and solution are similar, the long-term IVR dynamics differ. Elles et al. showed that vibrational quenching by the solvent dominates the long-term energy flow dynamics by removing energy from the molecule before the slower components of IVR are complete. As a result, quenching introduces an important kinetic limitation. The IVR cascade originating in the initially prepared state provides access to specific subsets of the full vibrational state density on a timedependent basis. Those states that are most strongly coupled to the initial state are populated first. When quenching limits the time available for IVR, weakly coupled energetic configurations populated late in the IVR cascade are never sampled. Therefore, the set of energetic configurations available to the reaction complex is not only non-statistical, but it is systematically biased toward those configurations most strongly coupled to the initial state.

Examples of mode- and bond-selective bimolecular reactions in the gas phase provide another point of comparison. Crim reviewed detailed studies of $\mathrm{C}-\mathrm{H}$ bond activation in bimolecular reactions where $\mathrm{Cl}$ abstracts an $\mathrm{H}$ or $\mathrm{D}$ atom from one of methane's vibrationally excited isotopologues. ${ }^{1}$ In all cases, $\mathrm{C}-\mathrm{H}$ or C-D stretching excitation leads to preferential cleavage of the vibrationally excited bond, but mode selective reactivity patterns are more subtle. In $\mathrm{CH}_{3} \mathrm{D}$, the symmetric $\mathrm{C}-\mathrm{H}$ stretching vibration is significantly more reactive than the slightly higher energy antisymmetric $\mathrm{C}-\mathrm{H}$ stretching state. Electronic structure calculations suggest that the approaching $\mathrm{Cl}$ atom perturbs methane's vibrations and leads to IVR in which the symmetric $\mathrm{C}-\mathrm{H}$ stretch evolves into a localized excitation of the $\mathrm{C}-\mathrm{H}$ bond pointing toward the $\mathrm{Cl}$ atom with the antisymmetric $\mathrm{C}-\mathrm{H}$ stretch evolving into excitation of the remaining $\mathrm{C}-\mathrm{H}$ bonds. Beck and co-workers note the relevance of these gas-phase studies to their orientationdependent and bond-selective studies of methane dissociation on $\mathrm{Ni}(100),{ }^{27} \mathrm{Ni}(110)$, and $\mathrm{Ni}(100) .{ }^{26}$

Aspects of surface reactivity studies parallel the processes at work in the gas phase and in solution. Experiments in which inelastic electron tunnelling or optical excitation of an adsorbed molecule prepares a superposition state are analogous to the gasphase and solution phase studies that prepare a zero-order superposition state. Following excitation, IVR in the initially prepared state begins immediately with relaxation into the doorway states. Quenching by the surface and limits on surface residence time will prevent IVR from reaching the full density of vibrational states available. The vibrational state energies and coupling patterns of a strongly chemisorbed adsorbate may differ significantly from the gas-phase molecule, and those differences must be accounted for when predicting IVR within a chemisorbed species. Weakly interacting adsorbates are most likely to have coupling patterns that parallel those of the gas phase molecule.

State-selected beam-surface experiments share similarities with gas phase, solution, and adsorbate excitation studies, but they also differ in important ways. When a molecule impinges on a metal surface, it encounters a bath of phonon 
and adsorbate-surface vibrations and vibrational state densities that can approach $10^{5}$ states $\mathrm{cm}^{-1}$ for a methane-surface complex. ${ }^{21}$ In solution, quenching limits IVR, but on a metal surface efficient quenching imposes an even stricter window on the time available for IVR, ${ }^{18,30}$ with vibrational lifetimes ranging from about 1 ps for $\mathrm{CO}$ chemisorbed on $\mathrm{Pt}(111)$ to more than $10 \mathrm{ps}$ for other small molecules adsorbed on metal surfaces. ${ }^{58,59}$ These lifetimes are shorter than, or comparable to those for the fastest IVR processes observed in large molecules. Surface residence time imposes another limit on the extent of IVR that can occur. Under single collision scattering conditions, sub-ps surface residence times are typical. The surface residence time of physisorbed molecules can be much longer, depending on the depth of the physisorption well and the surface temperature. ${ }^{60}$

The dynamics of energy redistribution also differ for beamsurface scattering, as detailed in Fig. 5. When the molecule is far from the surface (Fig. 5a), the rovibrational eigenstates $\left(\left\{\left|n_{\mathrm{a}}\right\rangle\right\}\right)$ of the system are those of the isolated molecule and surface. Narrow bandwidth laser excitation prepares a single initial state, $|s\rangle$, that is an eigenstate in the $\left\{\left|n_{\mathrm{a}}\right\rangle\right\}$ basis. There is no IVR and excitation remains in the initial state. When the molecule begins to experience the long-range attractive potential, molecule-surface interaction terms in the potential energy operator are no longer negligible (Fig. 5b), and a new set of vibrational eigenstates, $\left\{\left|n_{\mathrm{b}}\right\rangle\right\}$, describe the system. The initial state $|s\rangle$ is no longer an eigenstate in the $\left\{\left|n_{\mathrm{b}}\right\rangle\right\}$ basis - it must be written as a linear combination of the $\left\{\left|n_{\mathrm{b}}\right\rangle\right\}$. The heavy shading in Fig. $5 \mathrm{~b}$ illustrates the projection of $|s\rangle$ onto the $\left\{\left|n_{\mathrm{b}}\right\rangle\right\}$ basis. IVR occurs in this superposition state.

As the molecule continues to approach the surface (Fig. 5c), IVR in the superposition state causes the state's vibrational character to evolve in time. At the same time, the state's vibrational
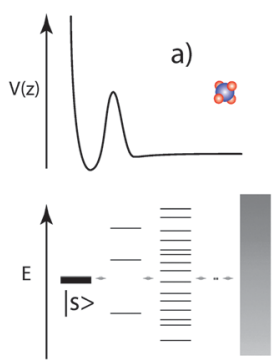
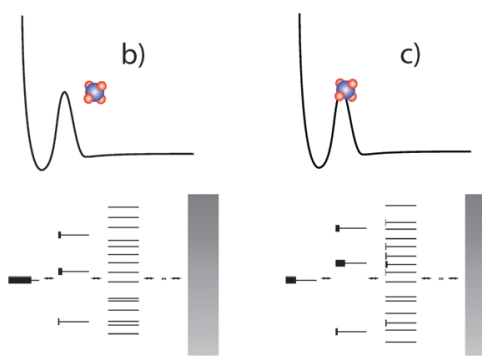

Fig. 5 Illustration of IVR during the molecule-surface encounter. In (a), the methane molecule is far from the surface. The tiered energy level diagram immediately below shows the vibrational eigenstates of the system for that molecule-surface geometry, the band on the right indicates the continuum of surface states. Heavy shading shows that the laser-prepared methane molecule is completely described by the single eigenstate $|s\rangle$. In (b), the molecule nears the barrier and experiences the gas-surface interaction potential. The energy levels shown immediately below (b) are the eigenstates of the system at this new molecule-surface distance. They differ from those shown in (a). Shading shows how the single eigenstate prepared in (a) projects onto the eigenstates of (b). As the molecule moves further toward the reaction barrier in (c), IVR occurs in the superposition state (b), and the eigenstates evolve further, resulting in the system's vibrational character being spread among even more eigenstates, as the shading shows. character is continually projected onto a new (and evolving) basis set of eigenstates, driving further IVR. When the molecule approaches the transition state, reaction will occur if and only if sufficient energy is localized along the reaction coordinate. If that does not occur, the molecule scatters non-reactively.

It is important to note that the identity of the doorway states for IVR depends on the initial state. For example, high frequency $\mathrm{C}-\mathrm{H}$ stretch fundamentals $(v=1)$ are most likely coupled to a small number of relatively high frequency hydride $($ e.g. $\mathrm{H}-\mathrm{C}-\mathrm{H})$ bending quanta. On the other hand, an excited bending vibration incident on the surface could couple to lower frequency bending or stretching motions that include two or more heavy atoms (e.g. $\mathrm{C}-\mathrm{C}-\mathrm{C}$ bends or $\mathrm{C}-\mathrm{C}$ stretches). Symmetry considerations also play a role in facilitating or restricting the coupling of zero-order states. Computational studies that reveal the rates and pathways of energy redistribution promise the most specific insight for a given molecule-surface system, but solution phase experiments suggest that a reagent's gas-phase coupling patterns can be a useful guide for predicting a molecule's propensity for mode- and bond-selective surface chemistry.

An important distinction between gas-surface reactivity studies and the cited examples of IVR in the gas-phase and solution is that reactivity studies explore IVR at energies at or above the barrier to reaction or diffusion. This brings the potential for much more significant chemical interaction between the molecule and metal surface while also providing insight into the rates and pathways of IVR in chemically reactive systems. Calculations reveal significant and mode-selective softening as the molecule approaches the surface. ${ }^{39,61,62}$ This behavior may tune or detune resonances between the initial state and the doorway states and impact the initial rate of IVR, but energy flow via loworder coupling into the doorway states will still dominate the initial stages of IVR. Recent high-level calculations of methane activation are consistent with this general propensity. For example, Nave and Jackson find little evidence for energy flow from the high frequency hydride stretch and bend modes of methane into low-frequency adsorbate-surface or phonon modes, ${ }^{39}$ and Mastromatteo and Jackson find that including overtone and combination states in their calculation has little impact on the dynamics of $\mathrm{C}-\mathrm{H}$ stretch excited methane on $\mathrm{Ni}(111) .{ }^{63}$ Jiang, Guo and co-workers report that a sudden vector projection model, which assumes no IVR during the reactive encounter, successfully captures the dominant dynamical features of methane activation. ${ }^{47,64,65}$

In all of these cases, surface-induced IVR will be restricted to initial energy redistribution into a small number of strongly coupled doorway states. The timescale for surface approach in hyperthermal beam-surface scattering is accelerated relative to the situation illustrated in Fig. 5. While potential gradients (and resulting forces on the nuclei) are large as the molecule approaches the barrier to reaction, there is little time for those forces to act on the nuclei and significantly alter vibrational motion.

In summary, studies of IVR in the gas phase and in solution point to "fast" IVR lifetimes on the picosecond timescale for a variety of polyatomic molecules whose vibrational state densities span nearly five orders of magnitude. The IVR rates appear 
to be predominantly set by the vibrational coupling patterns present in the isolated gas-phase molecules and not by overall vibrational state density. Solvents introduce quenching channels that compete kinetically with slower IVR processes, but they seem to have little influence on the initial rates of IVR in these molecules. Therefore, it appears that the rates of the fastest IVR processes are most sensitive to the identity of the target molecule and are relatively insensitive to the details of the molecule's chemical environment.

\section{Impact of IVR on surface reactivity and dynamics}

Quantum-state-resolved studies of gas-surface reactivity provide clear evidence for mode-and bond-selective chemistry and point to the limited extent of IVR during a reactive encounter. State-resolved studies of methane activation on $\mathrm{Ni}^{5}$ and $\mathrm{Pt}^{8,12}$ reveal that $\mathrm{C}-\mathrm{H}$ stretching excitation has a higher efficacy for promoting dissociative chemisorption than do bending states. This propensity for vibrational mode-selective reactivity has been predicted to extend to the dissociation of $\mathrm{H}_{2} \mathrm{O}$ on $\mathrm{Cu}(111){ }^{66}$ These observations show that IVR is not sufficiently fast to fully scramble the identity of the initially excited $\mathrm{C}-\mathrm{H}$ stretching and bending states prior to reaction. This is not surprising as stretch-bend coupling in methane is a third-order process between two distinct types of nuclear motion. Experiments also show that one quantum of $\mathrm{C}-\mathrm{H}$ stretch excitation leads to an extremely high degree of bond selectivity in tri-deutero methane, $\mathrm{CHD}_{3} .{ }^{13}$ Subsequent dissociative chemisorption measurements of methane isotopologues on $\operatorname{Pt}(110)-(1 \times 2)^{14}$ and simulations likewise reveal bond selective chemistry. The frequency mismatch between $\mathrm{C}-\mathrm{H}$ and $\mathrm{C}-\mathrm{D}$ oscillators leads to weak coupling between these oscillators and limited IVR during the brief gas-surface encounter.

Recently, Beck et al. have shown that mode-selective chemistry can occur even in reactions that proceed via a physisorbed precursor state. ${ }^{7}$ Vibrational state resolved studies of silane activation on $\mathrm{Si}(100)$ indicate that the two nearly degenerate $|1100\rangle$ and $|2000\rangle$ states differ significantly in their reactivity at low incident $E_{\text {trans }}$. Their results show that IVR is not complete even for reactions in which the gas-surface interaction time is significantly longer than is typical for a direct, single collision gas-surface interaction. They did not observe a precursormediated mode-selective enhancement under the conditions they investigated for $\mathrm{CH}_{4}$ dissociation on $\mathrm{Pt}(110)(1 \times 2)$.

One set of measurements point to conditions where IVR may be rapid enough to impact mode selectivity. State-resolved experiments show that the $\nu_{1}$ symmetric $^{9}$ and $\nu_{3}$ antisymmetric ${ }^{2} \mathrm{C}-\mathrm{H}$ stretching states of $\mathrm{CH}_{4}$ differ dramatically in their reactivity on $\mathrm{Ni}(100)$ even though the gas-phase identity of these states would appear to project similarly along the reaction coordinate. ${ }^{9}$ Several authors have proposed that the vibrationally adiabatic evolution of the $\mathrm{C}-\mathrm{H}$ stretching states as methane approaches the surface may result in differential transition state access. ${ }^{61,67,68}$ The $\nu_{1}$ symmetric $\mathrm{C}-\mathrm{H}$ stretching state correlates adiabatically with the single softened $\mathrm{C}-\mathrm{H}$ stretch of the bond nearest the surface while the $\nu_{3}$ antisymmetric stretch adiabatically correlates with excitation in the methyl group pointing away from the surface. For this scenario to explain the $\nu_{1}$ and $\nu_{3}$ data, IVR among the four $\mathrm{C}-\mathrm{H}$ oscillators in the molecule must be sufficiently rapid to permit the states to evolve adiabatically on the sub-picosecond timescale of the gas-surface interaction. In this case, the low coupling order (second order) between two $v=1 \mathrm{C}-\mathrm{H}$ stretching states and the inherently strong coupling among the individual $\mathrm{C}-\mathrm{H}$ oscillators in the gas-phase methane molecule could result in an IVR process fast enough to compete with the short gassurface encounter time. Recent full dimensional quantum dynamics calculations support the selective softening of the $\nu_{1}$ mode upon surface approach ${ }^{39,62}$ and highlight another effect that may contribute to the observed trend. When vibrationally non-adiabatic couplings are introduced, $\nu_{1}$ and $\nu_{3}$ differ in their ability to couple to lower energy vibrations, including the totally symmetric vibrational ground state, and thereby free vibrational energy for reaction coordinate motion.

While similar patterns of mode- and bond selectivity were observed for methane activation on surfaces and for the gas phase bimolecular reaction of $\mathrm{Cl}$ with methane or its isotopologues, ${ }^{69-71}$ the extent of bond-selectivity reported to date is greatest in gas-surface reactions. This most likely results from the extremely short surface residence times for methane, which severely limit the extent of IVR that can occur prior to the gasphase reagent's collision with the repulsive wall of the PES. Thus, gas-surface reactions, in which IVR is initiated by the collision and limited to the sub-ps duration of the gas-surface encounter, may provide the most favourable opportunity to extend mode- or bond-selective control to larger chemical systems at reactive energies.

These insights have important implications for computational studies of gas-surface reactivity, particularly for systems involving polyatomic reagents The extremely high vibrational state density at reactive energies poses a significant challenge to state-of-the-art theory. ${ }^{72}$ The propensities for vibrational energy flow observed in other chemical systems and highlighted here provide an additional validation for the many dynamical approximations currently required to study the gas-surface reactivity of polyatomic reagents.

The propensities for IVR discussed above also shed light on the potential role of surface phonon excitation in activating reactions. Calculations by Harrison and co-workers highlight the significant energy stored in the phonon bath at elevated surface temperatures. ${ }^{73}$ This energy was made available for redistribution within the reaction complex in their PC-MURT models. Our framework predicts that the high coupling order between the $\mathrm{ca} .200 \mathrm{~cm}^{-1}$ phonons and internal methane vibrations (1200-1400 $\mathrm{cm}^{-1}$ bends and 2900-3000 $\mathrm{cm}^{-1}$ stretches) results in slow IVR rates and limited energy exchange during the gas-surface encounter. Indeed, recent calculations show that lattice distortion resulting from surface phonon excitation promotes reactivity by directly exciting a motion that enhances transition state access, suggest that there is minimal energy exchange between the methane molecule and the active surface atom 
during the reactive encounter..$^{36,37,40,74,75}$ Another simulation suggests any enhancement of the reaction rate via phonons diminishes with increasing translational or vibrational energy of the incident methane. ${ }^{76}$ Recent experimental results support this theory ${ }^{77}$ and we plan to explore the role of phonon excitation on methane activation more fully in a future publication.

Inelastic tunnelling ${ }^{11,44,78}$ or infrared excitation studies of surface-adsorbed species ${ }^{79}$ differ in some ways from the beamsurface studies highlighted above, but key features of this framework still apply. The states available for excitation in these experiments are now eigenstates of the molecule surface complex, not those of an isolated gas-phase molecule. They are therefore more likely to include vibrational character from adsorbate-substrate and phonon modes. Because the initially prepared eigenstate is no longer a gas-phase eigenstate, IVR rates derived from gas-phase studies may be less helpful for predicting the mode- or bond-selective reactivity patterns. Line broadening of the adsorbate-substrate vibrations and excitation energy resolution limitations make it much more likely that the initially prepared state is a coherent superposition state that evolves in time. Nonetheless, IVR processes must still compete with quenching and reaction kinetics, and it is reasonable to expect that only those IVR pathways that rely on near-resonant, low-order coupling are fast enough to dominate vibrational energy redistribution on metal surfaces. Because vibrational quenching rates on metals are much faster than the "slow" IVR rates measured in gas-phase and solvated molecules of a similar vibrational state density, adsorbate vibrations will quench long before statistically sampling all energetic configurations in the microcanonical ensemble. When reactivity follows statistical predictions in these systems, it is because stochastic excitation and quenching processes lead to a statistical representation of initial states within the ensemble of reactants.

We expect the general conclusions of this work will extend from the model systems we cite to a wide range of thermally activated processes, including heterogeneous catalysis. Short surface residence times and rapid vibrational quenching are general features that will limit vibrational energy exchange during the molecule-surface encounter. Under these conditions, low-order coupling between nearly degenerate states will dominate energy redistribution during the molecule-surface encounter.

How does this affect surface reactivity? In many important surface reactions, the transition state is "late", and the reaction coordinate involves bond extension motions that project well onto higher frequency stretching vibrations in the reagent. At the same time, the vibrational bath of the system is dominated by states with multiquantum excitation of low frequency modes, including surface atom motion, frustrated translations, and rotations of the adsorbate, and low frequency skeletal and torsional vibrations of the molecular reagent. The large frequency mismatch between states with enhanced transition state access and those dominating the bath means that there will be very limited energy redistribution during a single moleculesurface encounter. The resulting picture of reactivity is one in which molecules approaching the surface in a vibrational state that provides favourable transition state access will dominate reactivity. Mode- and even bond-selective behavior will persist at this single collision level. Collisional vibrational energy transfer processes, including gas-phase collisions and vibrationally inelastic surface scattering, will replenish the population of the most reactive vibrational states and lead to an ensemble-averaged reactivity consistent with a statistical picture. While we expect high-frequency hydride stretches to be most decoupled from the low-frequency bath, relatively high frequency vibrations involving heavy atoms could also exhibit mode-selective chemistry if they are poorly coupled to other vibrations in the system. As the size and complexity of the chemical system increases, we expect a muting of nonstatistical behavior. Normal mode vibrations are less localized in larger molecules and project less well onto the reaction coordinate for breaking an individual bond. An increased vibrational state density enhances the chances for near-degenerate vibrational couplings that accelerate IVR, and the increased trapping probabilities and lifetimes for larger molecules will extend the time available for IVR during a single molecule-surface encounter. We are currently pursuing experiments to further investigate these effects.

\section{Conclusions}

This paper describes a phenomenological model for understanding the origin of mode- and bond-selective chemistry on surfaces, which increasingly seems to be the rule, rather than the exception for the reaction of small molecules on metal surfaces. Studies of vibrational energy flow and quenching in the gas phase, in solution, and on surfaces are combined to explain the observed reactivity of state-selected molecules in gas-surface reactions. A comparison of timescales reveals the kinetic processes most likely to impact reactivity. For beamsurface scattering studies, the gas-surface encounter initiates IVR and surface residence time severely limits the extent of IVR that can occur prior to reaction or non-reactive scattering. Timescales for vibrational quenching on metals and the desorption of physisorbed molecules are longer, but can still be much faster than the time required for IVR to completely and statistically redistribute energy within the reaction complex. These limitations ensure that molecules on the surface retain a memory of their initial vibrational identity and fulfill a necessary condition for mode- and bond-selective reactivity. The similarity of IVR rates for energy flow into and out of high-frequency vibrations in the gas and condensed phases suggest that propensities for mode-and bond-selective chemistry in the gas-phase may be useful predictors for analogous behavior on surfaces.

Of course, state-of-the-art computational studies will be required to assess quantitatively the behavior of individual chemical systems and vibrational states. The tiered model of IVR points out the sensitivity of initial IVR rates to the details of coupling in the molecule. Nonetheless, this framework identifies those features of the system that impact reactivity and serves as a useful guide for identifying surface reactions that are promising candidates for vibrationally mediated control of reaction yield and product identity. 


\section{Acknowledgements}

We thank the National Science Foundation for supporting this work through awards CHE-0809802 and CHE-1111702.

\section{Notes and references}

1 F. F. Crim, Proc. Natl. Acad. Sci. U. S. A., 2008, 105, 12654-12661.

2 L. B. F. Juurlink, P. R. McCabe, R. R. Smith, C. L. DiCologero and A. L. Utz, Phys. Rev. Lett., 1999, 83, 868-871.

3 M. P. Schmid, P. Maroni, R. D. Beck and T. R. Rizzo, J. Chem. Phys., 2002, 117, 8603-8606.

4 J. Higgins, A. Conjusteau, G. Scoles and S. L. Bernasek, J. Chem. Phys., 2001, 114, 5277-5283.

5 L. B. F. Juurlink, R. R. Smith, D. R. Killelea and A. L. Utz, Phys. Rev. Lett., 2005, 94, 208303.

6 R. R. Smith, D. R. Killelea, D. F. DelSesto and A. L. Utz, Science, 2004, 304, 992-995.

7 R. Bisson, T. T. Dang, M. Sacchi and R. D. Beck, J. Chem. Phys., 2008, 129, 081103.

8 R. Bisson, M. Sacchi, T. T. Dang, B. Yoder, P. Maroni and R. D. Beck, J. Phys. Chem. A, 2007, 111, 12679-12683.

9 P. Maroni, D. C. Papageorgopoulos, M. Sacchi, T. T. Dang, R. D. Beck and T. R. Rizzo, Phys. Rev. Lett., 2005, 94, 246104.

10 R. D. Beck, P. Maroni, D. C. Papageorgopoulos, T. T. Dang, M. P. Schmid and T. R. Rizzo, Science, 2003, 302, 98-100.

11 J. I. Pascual, N. Lorente, Z. Song, H. Conrad and H. P. Rust, Nature, 2003, 423, 525-528.

12 R. Bisson, M. Sacchi and R. D. Beck, Phys. Rev. B: Condens. Matter Mater. Phys., 2010, 82, 121404.

13 D. R. Killelea, V. L. Campbell, N. S. Shuman and A. L. Utz, Science, 2008, 319, 790-793.

14 L. Chen, H. Ueta, R. Bisson and R. D. Beck, Faraday Discuss. Chem. Soc., 2012, 157, 285-295.

15 A. L. Utz, Curr. Opin. Solid State Mater. Sci., 2009, 13, 4-12.

16 L. B. F. Juurlink, D. R. Killelea and A. L. Utz, Prog. Surf. Sci., 2009, 84, 69-134.

17 R. D. Beck and A. L. Utz, in Dynamics of Gas-Surface Interactions: Atomic-level Understanding of Scattering proceses at Surfaces, ed. R. D. Muiño and H. F. Busnego, SpringerVerlag, Berlin, 2013.

18 J. C. Tully, Annu. Rev. Phys. Chem., 2000, 51, 153-178.

19 V. A. Ukraintsev and I. Harrison, Surf. Sci., 1993, 286, L571-L576.

20 A. Bukoski, D. Blumling and I. Harrison, J. Chem. Phys., 2003, 118, 843-871.

21 H. L. Abbott, A. Bukoski and I. Harrison, J. Chem. Phys., 2004, 121, 3792-3810.

22 D. F. Kavulak, H. L. Abbott and I. Harrison, J. Phys. Chem. B, 2005, 109, 685-688.

23 H. L. Abbott and I. Harrison, J. Phys. Chem. C, 2007, 111, 13137-13148.

24 P. J. Robinson and K. A. Holbrook, Unimolecular Reactions, Wiley Interscience, New York, 1972.

25 K. Freed, Faraday Discuss. Chem. Soc., 1979, 67, 231-235.
26 B. L. Yoder, R. Bisson, P. M. Hundt and R. D. Beck, J. Chem. Phys., 2011, 135, 224703.

27 B. L. Yoder, R. Bisson and R. D. Beck, Science, 2010, 329, 553-556.

28 N. Chen, Y. Huang and A. L. Utz, J. Phys. Chem. A, 2013, 117, 6250-6255.

29 I. Rahinov, R. Cooper, D. Matsiev, C. Bartels, D. J. Auerbach and A. M. Wodtke, Phys. Chem. Chem. Phys., 2011, 13, 12680-12692.

30 A. M. Wodtke, D. Matsiev and D. J. Auerbach, Prog. Surf. Sci., 2008, 83, 167-214.

31 J. C. Polanyi, Acc. Chem. Res., 1972, 5, 161-168.

32 S. Yan, Y. T. Wu, B. L. Zhang, X. F. Yue and K. P. Liu, Science, 2007, 316, 1723-1726.

33 G. Herzberg, Molecular Spectra and Molecular Structure II. Infrared and Raman Spectra of Polyatomic Molecules, Van Nostrand Reinhold, New York, 1945.

34 E. Venuti, L. Halonen and R. G. Della Valle, J. Chem. Phys., 1999, 110, 7339-7347.

35 G. Henkelman, A. Arnaldsson and H. Jonsson, J. Chem. Phys., 2006, 124, 044706.

36 S. Nave and B. Jackson, J. Chem. Phys., 2007, 127, 224702.

37 S. Nave and B. Jackson, J. Chem. Phys., 2009, 130, 054701.

38 S. Nave, A. K. Tiwari and B. Jackson, J. Chem. Phys., 2010, 132, 054705.

39 S. Nave and B. Jackson, Phys. Rev. B: Condens. Matter Mater. Phys., 2010, 81, 233408.

40 A. K. Tiwari, S. Nave and B. Jackson, Phys. Rev. Lett, 2009, 103, 253201.

41 M. P. Schmid, P. Maroni, R. D. Beck and T. R. Rizzo, Rev. Sci. Instrum., 2003, 74, 4110-4120.

42 P. R. McCabe, L. B. F. Juurlink and A. L. Utz, Rev. Sci. Instrum., 2000, 71, 42-53.

43 P. Maroni, D. Papageorgopoulos, A. Ruf, R. D. Beck and T. R. Rizzo, Rev. Sci. Instrum., 2006, 77, 054103.

44 Y. Sainoo, Y. Kim, T. Okawa, T. Komeda, H. Shigekawa and M. Kawai, Phys. Rev. Lett., 2005, 95, 246102.

45 B. Jackson, in Dynamics of Gas-Surface Interactions: Atomiclevel Understanding of Scattering proceses at Surfaces, ed. R. D. Muiño and H. F. Busnego, Springer-Verlag, Berlin, 2013.

46 B. Jackson and S. Nave, J. Chem. Phys., 2011, 135, 114701.

47 B. Jiang, R. Liu, J. Li, D. Xie, M. Yang and H. Guo, Chem. Sci., 2013, 4, 3249-3254.

48 C. G. Elles and F. F. Crim, Annu. Rev. Phys. Chem., 2006, 57, 273-302.

49 C. G. Elles, M. J. Cox and F. F. Crim, J. Chem. Phys., 2004, 120, 6973-6979.

50 E. L. Sibert III, J. T. Hynes and W. P. Reinhardt, J. Chem. Phys., 1984, 81, 1135-1144.

51 P. R. Stannard and W. M. Gelbart, J. Phys. Chem., 1981, 85, 3592-3599.

52 Coupling strength is greatest when the interacting states are nearly degenerate and their coupling order (defined as the number of vibrational quanta that must be changed to convert one state into the other) is low. 
53 K. K. Lehmann, B. H. Pate and G. Scoles, J. Chem. Phys, 1990, 93, 2152-2153.

54 R. S. von Benten, Y. Liu and B. Abel, J. Phys. Chem. A, 2010, 114, 11522-11528.

55 H. S. Yoo, D. A. McWhorter and B. H. Pate, J. Phys. Chem. A, 2004, 108, 1380-1387.

56 H. S. Yoo, M. J. DeWitt and B. H. Pate, J. Phys. Chem. A, 2004, 108, 1365-1379.

57 H. S. Yoo, M. J. DeWitt and B. H. Pate, J. Phys. Chem. A, 2004, 108, 1348-1364.

58 J. D. Beckerle, R. R. Cavanagh, M. P. Casassa, E. J. Heilweil and J. C. Stephenson, J. Chem. Phys., 1991, 95, 5403-5418.

59 V. Krishna and J. C. Tully, J. Chem. Phys., 2006, 125, 054706.

60 G. O. Sitz and C. B. Mullins, J. Phys. Chem. B, 2002, 106, 8349-8353.

61 R. Milot and A. P. J. Jansen, Phys. Rev. B: Condens. Matter Mater. Phys., 2000, 61, 15657-15660.

62 K. G. Prasanna, R. A. Olsen, A. Valdes and G.-J. Kroes, Phys. Chem. Chem. Phys., 2010, 12, 7654-7661.

63 M. Mastromatteo and B. Jackson, J. Chem. Phys., 2013, accepted for publication.

64 B. Jiang and H. Guo, J. Chem. Phys., 2013, 138, 234104.

65 B. Jiang and H. Guo, J. Phys. Chem. C, 2013, 117, 16127-16135.
66 B. Jiang, D. Xie and H. Guo, Chem. Sci., 2013, 4, 503-508.

67 L. Halonen, S. L. Bernasek and D. J. Nesbitt, J. Chem. Phys., 2001, 115, 5611-5619.

68 G. P. Krishnamohan, R. A. Olsen, G. J. Kroes, F. Gatti and S. Woittequand, J. Chem. Phys., 2010, 133, 144308.

69 H. A. Bechtel, J. P. Camden, D. J. A. Brown and R. N. Zare, J. Chem. Phys., 2004, 120, 5096-5103.

70 Z. H. Kim, H. A. Bechtel and R. N. Zare, J. Am. Chem. Soc., 2001, 123, 12714-12715.

71 C. J. Annesley, A. E. Berke and F. F. Crim, J. Phys. Chem. A, 2008, 112, 9448-9453.

72 G.-J. Kroes, Phys. Chem. Chem. Phys., 2012, 14, 14966-14981.

73 A. Bukoski and I. Harrison, J. Chem. Phys., 2003, 118, 9762-9768.

74 S. Nave and B. Jackson, Phys. Rev. Lett., 2007, 98, 173003.

75 A. K. Tiwari, S. Nave and B. Jackson, J. Chem. Phys., 2010, 132, 134702.

76 M. Moix Teixidor and F. Huarte-Larranaga, Chem. Phys., 2012, 399, 264-271.

77 D. R. Killelea, V. L. Campbell, N. S. Shuman, R. R. Smith and A. L. Utz, J. Phys. Chem. C, 2009, 113, 20618-20622.

78 W. Ho, J. Chem. Phys., 2002, 117, 11033-11061.

79 B. Redlich, H. Zacharias, G. Meijer and G. von Helden, J. Chem. Phys., 2006, 124, 044704. 